\title{
Compositional simulation of a refinery coker furnace- An industrial example of two-phase flow with chemical reaction
}

\author{
SIGURD SKOGESTAD and TRULS GUNDERSEN† \\ and \\ ODDVAR JOHNSEN $\ddagger$
}

Keywords: Simulation, numerical integration, phase equilibria, coke deposition, two-phase flow

A computer program (KOKSOVN) has been developed for compositional steady state simulation of a refinery delayed coker furnace. The main objective of this work has been to establish a tool for studying the effects that influence the deposition of coke on the inside walls of the tubes in order to maximize the time of operation (cycle time) between each cleaning of the tubes with a resulting stop in production.

The program basically consists of a standard integration package which steps along the reactor (or pipeline) while solving the vapour-liquid equilibrium (VLE) and estimating physical properties for each step.

Using a modular approach in the development, the resulting computer program has some general features which make it a possible simulation tool for any non-adiabatic plug flow reactor with two-phase flow. Depending on the chemical system, the routines for thermophysical and transport properties, phase equilibria and chemical reaction may be replaced by other methods.

The program may also be used to simulate a pipeline with one or two-phase flow. Since, however, the total composition in this case is constant, it would probably be more efficient to use tables based on the pressure values, instead of performing tedious VLE calculations along the pipeline as is done in the present program.

Scope

Modeling a delayed coker furnace involves a large variety of physical phenomena such as heat transfer, chemical reaction, phase equilibria and two-phase flow. The furnace is thus a very complex 'unit operation'. The mathematical model involves a set of coupled ordinary differential equations and a set of algebraic equations (some of which are implicit and thus require iteration), which have to be solved simultaneously. The pressure and the concentration of the key components of the chemical reactions are the state variables.

The algebraic equations are solved by performing an isenthalpic VLE calculation at each integration step in order to find the temperature and the phase split, which are the basic variables in the determination of the transport properties and the rates of reaction for the cracking that takes place.

The operating parameters include inlet temperature and pressure, feed rate, furnace heat duty (distributed as a known heat flux profile), and steam injection. Another objective has been to study the effects of alternative feedstocks to the unit. This makes feed composition an important parameter and a proper characterization of the feed crucial.

The computer program employs the Hindmarsh-LSODE algorithm for numerical solution of the ordinary differential equations and uses state-of-the-art methods for two-phase flow and phase equilibria. The total pressure drop in the

Received 26 November 1985.

$\dagger$ Norsk Hydro, Porsgrunn Fabrikker, N-3901 Porsgrunn, Norway.

\$ Rafinor, Norway. 
heater (about 20 bars) consists of gravitation, acceleration and friction, the latter being modeled by HTFS methods. The VLE is modeled by the cubic RKS equation of state.

Conclusions and significance

Because of the rigorous approach taken in the modeling, the computer program has proven to be predictive for cases where a complete characterization of the feed to the heater has been available. The predicted pressure drop through the furnace and the temperature profile match plant data very well.

To study rigorously how alternative feedstocks to the delayed coker section affects the coker furnace, the model has to be extended to include the coker drum and the bottom of the distillation column. Since such models were not available, the feed to the furnace (characterized by its TBP curve) was estimated based on the TBP data of the feed to the column. This did not turn out to be completely satisfactory.

The information obtained by using the simulation model has made it possible to run the actual unit in a more optimal way by reducing the coke deposition on the inner walls of the tubes and thus increasing the cycle times for the plant. One important spin-off from the model has been the increased general understanding of the delayed coker furnace, which is the case with most mechanistic process simulation models.

The computer time for each simulation is far from prohibitive, although the unit (furnace) is complex and has been rigorously modeled. A typical case where input pressure and furnace heat duty are varied to match output pressure and temperature takes about 4 minutes CPU time on a UNIVAC-1100/62. To tackle the situation described, a two-dimensional Newton-Raphson iteration has to be applied, with typically 8 calculations of the reactor.

\section{Introduction}

This paper presents a steady state simulation model developed by Norsk Hydro a.s for a delayed coker furnace at Rafinor's Mongstad refinery. Due to coke deposition on the inner walls of the tubes in the furnace, the operating company had problems with short cycle times. The thin coke layer increases the pressure drop through the furnace and leads to high wall temperatures which may lead to material problems as well as low furnace energy economy.

The coke formation is inevitable; after all this is a delayed coker unit. Ideally, the furnace should be able to operate from half a year up to one year, depending on the quality of the feed, between each shutdown of the plant for decoking. For the actual coker unit at Mongstad, however, cycle times down to only three months were experienced. The economic consequences of the reduced cycle times can be substantial, depending on the product prices in the market.

The main objective of this work has therefore been to develop a rigorous simulation model of the delayed coker furnace which may be used to guide the operating company in finding optimal operating conditions for the unit. This essentially means running the unit in a way that reduces the coke deposition on the inner walls of the tubes. Operating parameters include inlet temperature, feed rate, amount of fuel (heat flux profile), and steam rate with corresponding injection point.

The complexity of the unit excludes the use of general purpose flowsheet simulators like PROCESS (SIMSCI 1983), DESIGN II (Chemshare 1981) or ASPEN PLUS (Aspen Tech 1983). Special purpose programs have been developed by consulting companies, but these are not commercially available. Because of this, it was decided to develop a new simulation program while using as much as possible of existing software within the company.

Since the partial vaporization of the liquid feed in the furnace plays an important role for the flow conditions and chemical reactions taking place, it was decided 
at an early stage to model the VLE as accurately as possible and thus use a compositional model. It was also decided to use a tubular reactor model (resulting in a set of coupled ordinary differential equations), rather than a series of continuous stirred tank reactors (CSTR). One major concern regarding the compositional approach, was the computer time involved in solving a rigorous VLE at each integration step in the furnace.

\section{General features of the program}

Before discussing this particular problem in detail, a more general approach to the problem is of interest. Essentially, the problem is modeling and simulation of a two-phase non-adiabatic tubular reactor. Obviously, this is a generalization of the more common two-phase flow pipeline and the single phase tubular reactor. The governing equations are nonlinear ordinary differential equations (ODE's) and nonlinear algebraic equations. An entirely rigorous approach would include partial differential equations, but these may be converted with little loss of accuracy to ODE's by using lumped values (averaging in the radial direction).

The number of key variables in the problem is equal to the number of independent differential equations. In general this will be equal to $n_{\mathrm{r}}+2$, where $n_{\mathrm{r}}$ is the number of independent chemical reactions, and the two other state variables are pressure and enthalpy.

The most important algebraic equations are those involved in the isenthalpic flash to find the temperature and phase distribution based on composition and pressure. The most direct approach is to solve these equations at each step of the integration. From a computational point of view, this may be very costly and for 'stiff' problems may even become prohibitive.

An alternative approach is to generate, before the integration starts, a table relating temperature, phase split, liquid and vapour composition, and physical properties to the state variables. In the simple case of two-phase flow with no chemical reaction, this table will be two-dimensional and such an approach is very attractive. As the number of chemical reactions increases, the dimension of the table and the work involved in creating and interpolating in the table increase significantly and the approach is no longer competitive with the direct VLE calculation approach.

In many cases, boundary conditions are given at the outlet of the reactor instead of the inlet, resulting in a two-point boundary value problem. This may be solved by using methods especially developed for such problems (e.g. collocation methods), or by iteratively solving initial value problems ('shooting') with different inlet conditions.

\section{Description of the process}

The coker furnace is part of a delayed coker unit which upgrades residual oil fractions to petroleum coke and lighter fractions. While petroleum coke in earlier years was regarded as a mere by-product (referred to as Green Coke and sold as low grade fuel), the new applications of upgraded (calcined) coke, mainly in the manufacture of carbon electrodes for the aluminium industry, have made it a valuable primary product for the refinery industry.

\section{The delayed coking section}

A good introduction to delayed coking is given by Fletcher (1983) in a paper discussing some of the operating problems that initiated this simulation model. A 


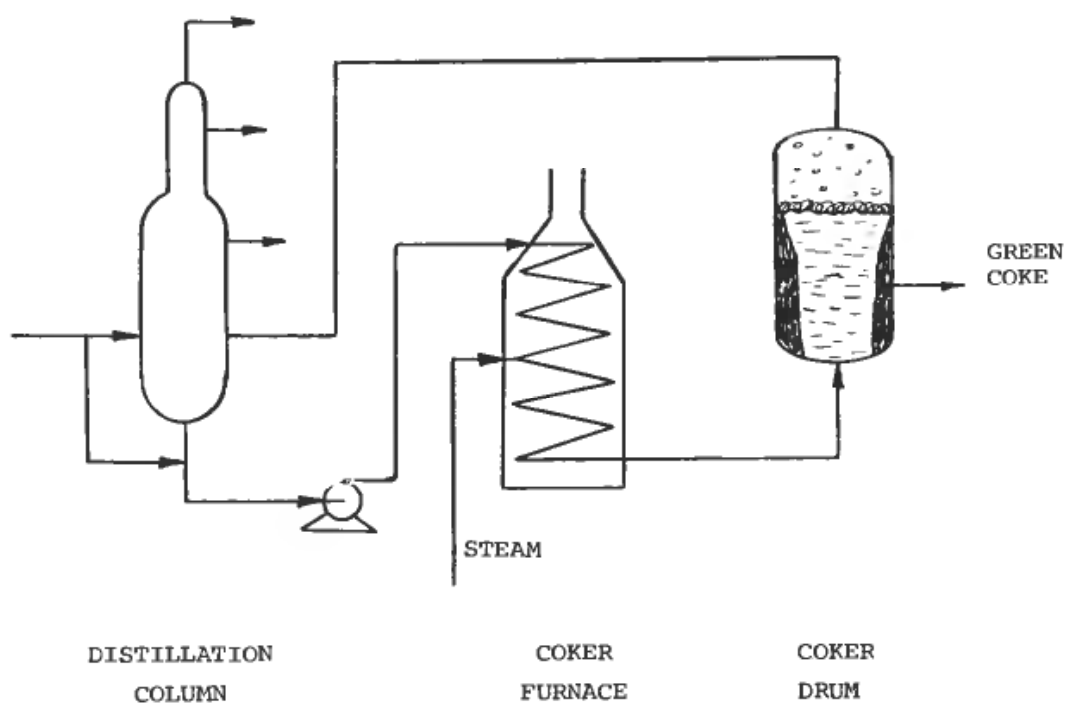

Figure 1. Delayed coker section with column, furnace and drum.

simplified flowsheet of the process is given in Fig. 1, showing the fractionation column, the furnace and the coke drum. Although the simulation model only includes the coker furnace, it is of interest to give a brief introduction to the whole coker section.

Delayed coking is a semi-continuous process since the actual coke formation is achieved in two batch-wise coke drums, working one at a time. While one drum is on-stream being filled with coke (takes about 24 hours), the other is taken out of the production line, and so called Green Coke is removed. The drum decoking involves steaming, quenching with water, hydraulic decoking and rewarming with hot vapours.

The fresh feed enters the coker section through the fractionation column in Fig. 1 , where it is mixed with recycled heavy gas oil from the coke drum. The base section of this column acts as a feed vessel to the coker furnace. The bottom stream from the column is then pumped to the coker furnace.

The prime objective of the coker furnace is to heat up the bottom stream from the column as quickly as possible before the process stream enters the coke drum. The inlet pressure should be able to maintain high velocities in the furnace where the pressure drop is typically in the range of 20 bars. The vapour/liquid stream from the furnace enters the coke drum where the liquid boils until it is thermally cracked to gas products and a later polymerization to coke which deposits on the walls and gradually fills the drum.

\section{The coker furnace}

In the coker furnace, the process stream enters in the convection section at the top of the furnace and flows through 55 horizontal tubes separated by bends down through the radiation section and finally leaves at the bottom of the furnace. Each tube is about $20 \mathrm{~m}$ long giving a total reactor length of more than $1000 \mathrm{~m}$ and a liquid residence time of about 2 minutes. The coker furnace heats the process stream, and the cracking reactions are supposed to take place in the drum, but it is of course inevitable that some cracking occurs in the furnace itself. Approximately 
$10 \mathrm{wt} \%$ of the feed is cracked in the furnace to lighter components $\left(\mathrm{C}_{1}\right.$ to $\left.\mathrm{C}_{14}\right)$ and to heavier polycyclic aromatics which provide the first step in the coke formation process.

As a result, coke deposits on the inner walls of the tubes, resulting in increased pressure drop and higher tube wall temperatures because of the additional heat transfer resistance through the thin coke layer. These effects eventually lead to shutdown of the unit, because the pump may no longer be able to provide the necessary inlet pressure, the heating becomes uneconomical, or the high wall temperatures result in material problems.

Although the cracking rates increase with temperature, coke deposition is usually not experienced at the end of the furnace, where the temperature is highest. Rather, the problem occurs at some intermediate part of the furnace, where the velocities are lower than at the very end of the furnace.

The cycle time between shutdowns of the coker section due to coke deposition in the furnace is therefore a crucial parameter for the economy of the process, and several design considerations are aimed at lowering this deposition. Some of the important aspects of the furnace operation are short residence times, high heat transfer rates in the radiant section, good distribution of heat in the fire box and high tube velocity or turbulence.

At the Mongstad refinery, steam injection is applied to increase tube velocity in order to reduce coke deposition in the tubes. As a result, partly due to the steam injection itself and partly due to additional vaporization of liquid, the linear velocity increases from about $3 \mathrm{~m} / \mathrm{s}$ to $6 \mathrm{~m} / \mathrm{s}$ at the injection point. The linear velocity at the outlet is about $40 \mathrm{~m} / \mathrm{s}$.

The coker furnace is similar to a visbreaker. In a visbreaker the outlet stream is returned directly to the column instead of going to the drum.

\section{Physical phenomena in the furnace}

To obtain a rigorous model of the furnace, the following physical phenomena must be included in the model:

* Phase equilibria (VLE)

* Two-phase flow (pressure drop)

* Chemical reaction

* Heat transfer

* Coke deposition

Sub-cooled liquid at high pressure enters the furnace and is gradually heated to its boiling point. The specific point in the furnace where the separate vapour phase is formed almost coincides with the injection of the steam, as indicated in Fig. 2. The length of the reactor (about $1000 \mathrm{~m}$ ) and its small inner diameter (about $0.1 \mathrm{~m}$ ), result in a pressure drop of nearly 20 bars. It is thus of extreme importance to describe the flow through the furnace, and since some of the liquid is vaporized, we have a two-phase flow problem.

Most of the pressure drop is due to the two-phase friction pressure drop and it should be handled rigorously. The increase of velocity through the furnace results in a significant acceleration pressure drop (representing $10 \%$ of the total pressure drop at the end of the reactor). Pressure changes due to gravitation are less important, since the elevation difference between inlet and outlet is only $10 \mathrm{~m}$. 


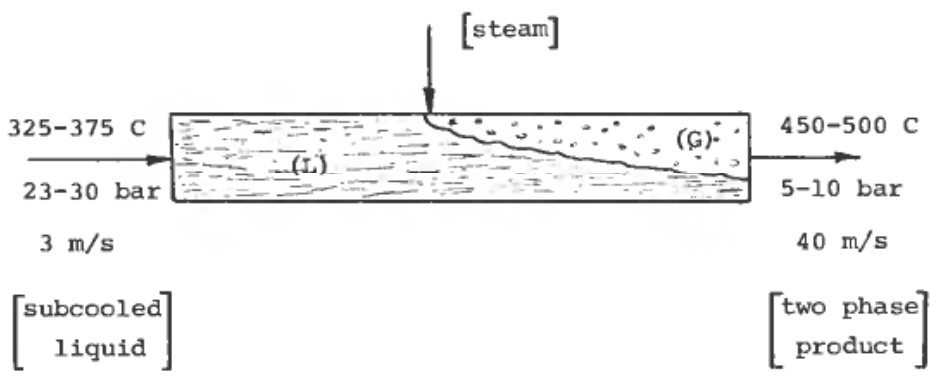

Figure 2. Schematic drawing of the tubes of the furnace.

The VLE is obviously important for estimating the phase-split and velocities to be used for the two-phase pressure drop calculations, but it is also crucial for estimating the temperature profile and liquid composition used to estimate the rate of thermal cracking reactions. The chemical reactions are endothermic and counteract the temperature increase resulting from the heating.

The geometry of the furnace is complex, and an accurate description of the heat transfer by radiation and convection on the fire side of the furnace will add another dimension to the complexity of the simulation. However, since the major resistance to heat transfer is on the fire side of the tubes, the process conditions have little influence on the heat transfer. The heat flux profile may thus be regarded as known for simulating the process side of the furnace. Heat flux profiles may be obtained from plant data or from fire box simulations.

The coke deposition should be modeled as a steady state between the following two processes:

* 'Chemical' deposition of coke on the inner walls of the tubes, controlled by an activation energy.

* Physical removal of this coke layer, controled by the flow conditions in the tubes (velocity and degree of turbulence in the tubes).

The relative importance of the described phenomena (two-phase flow, VLE, chemical reaction, heat transfer and coke deposition) depends on the actual objective of the simulation model. Since originally accurate models for the pressure drop and temperature profile were identified as important in attempting to find more optimal ways of running the unit, the VLE becomes crucial. The resulting phase distribution is important for the two-phase pressure drop calculations, and the temperature is a result of an isenthalpic flash calculation.

If the ability to describe the effects of alternative feedstocks to the coker unit had been the main objective, the knowledge of stoichiometry and kinetics of the cracking reactions would have been more important for the simulation model. In this case more attention would also have to be given to a proper characterization procedure, and it might have been necessary to include the recycle stream from the drum which mixes with the pure feed in the base of the fractionation column.

The above discussion makes it clear that the delayed coker furnace (when modeled as a non-adiabatic tubular reactor with two-phase flow) is a very general chemical engineering 'unit operation', where many physical phenomena take place and interact to give a complex total picture. Using such a general approach to solve a specific problem has the advantageous spin-off that the resulting computer program has features which may be applied to other problems as well. 


\section{Mathematical model}

The coker furnace is modeled as a non-adiabatic plug-flow reactor with twophase flow. The conservation equations for mass, heat and momentum result in a set of coupled ordinary differential equations. In addition, there is a set of algebraic equations (some of which are implicit and must be solved by iteration) which have to be solved simultaneously with the differential equations.

\section{Material balance}

The heavy fractions of the feed (modeled as hypothetical or pseudo components) will crack into lighter hydrocarbons $\left(\mathrm{C}_{1}\right.$ to $\left.\mathrm{C}_{14}\right)$ and heavier polycyclic aromatics because of the high temperature in the furnace. These reactions are assumed to take place in the liquid phase with first order kinetics. The minimum number of differential equations needed to model the material balance is equal to the number of independent reactions, which in this case is equal to the number of pseudo components. The material balances for these heavy fractions are given by Eq. 1 .

$$
\frac{d F_{\mathrm{i}}}{d z}=-r_{\mathrm{i}} \cdot A_{\text {liq }} \text { for } i=1, n_{\text {hyp }}
$$

where

$F_{\mathrm{i}}=$ molar flow rate of component number i $[\mathrm{kmol} / \mathrm{s}]$

$z \quad=$ axial coordinate of the reactor [m]

$r_{\mathrm{i}}=$ rate of cracking for reaction $\mathrm{i}\left[\mathrm{kmol} / \mathrm{s} \cdot \mathrm{m}^{3}\right]$

$A_{\text {liq }}=$ liquid void fraction in the tube $\left[\mathrm{m}^{2}\right]$

$n_{\text {hyp }}=$ total number of hypothetical components in the feed

The rates of reaction depend on temperature and mole fraction of the pseudo components. The material balance for all other components are obtained from simple stoichiometric relations.

\section{Enthalpy balance}

The enthalpy of the fluid will vary through the furnace as a result of the heating. The heat transfer will have contributions from both radiation and convection, and the complex geometry of the furnace makes rigorous modeling of this phenomenon very difficult. In the simple case, where only convective heat transfer is assumed, the enthalpy of the fluid will change according to the following equation:

$$
\frac{d H}{d z}=U \cdot\left(T_{\mathrm{o}}-T\right) \cdot \pi \cdot D
$$

where

$H=$ enthalpy of the fluid $[\mathrm{kW}]$

$U=$ overall heat transfer coefficient $\left[\mathrm{kW} / \mathrm{K} \cdot \mathrm{m}^{2}\right]$

$T_{\mathrm{o}}=$ temperature outside the tubes $[\mathrm{K}]$

$T=$ temperature inside the tubes $[\mathrm{K}]$

$D=$ inner tube diameter $[\mathrm{m}]$

The temperature difference between the process side and the outside of the tube walls is less than $\frac{1}{10}$ of the total temperature drop. Consequently, the dominating 
heat transfer resistance is on the fire side of the tubes, and there is little reason to combine fire box simulations with the simulation of the behaviour inside the tubes. The heat flux profile may be obtained by a separate simulation or by measurements along the tubes. The differential equation for the enthalpy balance is eliminated, and the simplified enthalpy balance equation then becomes:

$$
H(z)=H(0)+\int_{0}^{z} Q(z) \cdot \pi \cdot D \cdot d z
$$

where

$$
\begin{aligned}
& H(z)=\text { enthalpy of fluid at position } z[\mathrm{~kW}] \\
& H(0)=\text { enthalpy at furnace inlet }[\mathrm{kW}] \\
& Q(z)=\text { heat flux at position } z\left[\mathrm{~kW} / \mathrm{m}^{2}\right]
\end{aligned}
$$

Note that a heat of reaction term is not included since the same reference state (combustion products) is used for all components. When the total enthalpy of the stream is known, and composition and pressure are given from the other differential equations, the temperature is found from an isenthalpic flash calculation.

\section{Momentum balance}

Friction, acceleration and gravitation terms contribute to the two-phase pressure drop in the furnace, as written in the following overall equation (see Butterworth and Hewitt (1977)):

where

$$
\left[\frac{d P}{d z}\right]=\left[\frac{d P}{d z}\right]_{\mathrm{f}}+\left[\frac{d P}{d z}\right]_{\mathrm{a}}+\left[\frac{d P}{d z}\right]_{\mathrm{g}}
$$

$P=$ pressure at position $z$ [bar $]$

$z=$ axial coordinate in reactor $[\mathrm{m}]$

$f=$ subscript for friction

$a=$ subscript for acceleration

$g=$ subscript for gravitation

The acceleration pressure drop (due to change in kinetic energy) is given by:

$$
\begin{gathered}
{\left[\frac{d P}{d z}\right]_{\mathrm{a}}=-G^{2} \cdot \frac{d b}{d z}} \\
b=\frac{(1-x)^{2}}{\rho_{\mathrm{L}} \cdot(1-\alpha)}+\frac{x^{2}}{\rho_{\mathrm{G}} \cdot \alpha}
\end{gathered}
$$

where

$G=\operatorname{mass}$ flux $\left[\mathrm{kg} / \mathrm{m}^{2} \cdot \mathrm{s}\right]$

$x=$ weight fraction vapour ('quality')

$\alpha=$ cross section in the tubes occupied by vapour ('void fraction')

$\rho_{L}=$ liquid density $\left[\mathrm{kg} / \mathrm{m}^{3}\right]$

$\rho_{G}=$ vapour density $\left[\mathrm{kg} / \mathrm{m}^{3}\right]$

Equation (5) may be written as a function of the state variables $F_{\mathrm{i}}, H$ and $P$ :

$$
\left[\frac{d P}{d z}\right]_{\mathrm{a}}=-G^{2} \cdot\left[\sum_{i=1}^{n_{\mathrm{hpp}}} \frac{\partial b}{\partial F_{\mathrm{i}}} \cdot \frac{d F_{\mathrm{i}}}{d z}+\frac{\partial b}{\partial H} \cdot \frac{d H}{d z}+\frac{\partial b}{\partial P} \cdot \frac{d P}{d z}\right]
$$


Calculating the acceleration term according to equation (7) will therefore involve expressions for $\partial x / \partial F_{\mathrm{i}}, \partial \alpha / \partial F_{\mathrm{i}}, \partial \rho_{\mathrm{L}} / \partial F_{\mathrm{i}}$, etc. This is obviously much too complicated, as it would require analytical or numerical derivatives for the flash equations, density correlations, etc. with respect to the state variables. The acceleration pressure drop is thus obtained by numerical differentiation of the parameter $b$ with respect to the reactor length:

$$
\left[\frac{d P}{d z}\right]_{\mathrm{a}}=-G^{2} \cdot \frac{\left(b_{2}-b_{1}\right)}{\left(z_{2}-z_{1}\right)}
$$

The points $\left(z_{1}, b_{1}\right)$ and $\left(z_{2}, b_{2}\right)$ should ideally be separated only as much as the numerical accuracy of the computer requires in order to avoid round-off errors becoming important, and so that the approximation of the tangent is as good as possible. In order to avoid introducing excessive calculations to improve the acceleration pressure drop only slightly, the points adopted in the simulation program presented here are the ones chosen by the numerical differential equation solver (LSODE).

In the first part of the reactor, these points are several tube lengths apart, while near the reactor outlet, LSODE has to slow down, and the points are much closer. This coincides nicely with the fact that the acceleration pressure drop only accounts for $0.1 \%$ of the total pressure drop at the inlet, while it accounts for about $10 \%$ at the outlet.

The friction pressure drop is considerable in the furnace, and state-of-the-art methods from HTFS (Whalley 1981) are applied in the simulation program. These methods are available for member companies only, but the overall equation in the two-phase flow situation is:

$$
\Delta P_{\mathrm{f}}=\Delta P_{\mathrm{L}}+C_{\mathrm{c}} \cdot\left[\Delta P_{\mathrm{L}} \cdot \Delta P_{\mathrm{G}}\right]^{1 / 2}+\Delta P_{\mathrm{G}}
$$

where

$\Delta P_{\mathrm{f}} \quad=$ total friction pressure drop.

$\Delta P_{\mathrm{L}, \mathrm{G}}=$ single phase friction pressure drop for liquid and vapour if these phases had been alone in the tube.

$C_{\mathrm{c}} \quad=$ function of $G, D, \varepsilon, \alpha, x, \rho_{\mathrm{L}}, \rho_{\mathrm{G}}, \mu_{\mathrm{L}}$ and $\mu_{\mathrm{G}}$ (obtained by HTFS methods).

The key parameter $C_{\mathrm{c}}$ is a complex function of 'quality' and 'void fraction' as well as transport properties like density and viscosity for vapour and liquid.

Finally, the gravitation term may be obtained from Eq. 10 which is formulated as pressure drop (decrease in pressure). Since, for the actual case, elevation decreases ( $d y / d z$ is negative), the gravitation term gives a positive contribution to the pressure of the fluid.

$$
\left[\frac{d P}{d z}\right]_{\mathrm{g}}=\left[\rho_{\mathrm{L}} \cdot(1-\alpha)+\rho_{\mathrm{G}} \cdot \alpha\right] \cdot g \cdot \frac{d y}{d z}
$$

where

$g=$ acceleration of gravity $\left[\mathrm{m} / \mathrm{s}^{2}\right]$
$y=$ vertical coordinate (level) $[\mathrm{m}]$ 


\section{Chemical reaction}

Since the cracking reactions taking place in the furnace are very complex and do not constitute the primary phenomenon in the unit, it was decided to use simplified models with overall reactions and first order kinetics. After all, the furnace is primarily a heater and not a reactor. The main objective when modeling these reactions is thus to give a reasonable overall composition of the mixture, in order to calculate the VLE properly.

The feed (given as a TBP curve) is broken down into $n_{\text {hyp }}$ pseudo components (typically 5) which will react (thermal cracking) into coke and lighter components $\left(\mathrm{H}_{2}, \mathrm{CH}_{4}, \mathrm{C}_{2}, \mathrm{C}_{3}\right.$, etc.) through a first order reaction. This assumption has proven to give reasonable results for the furnace, but when modeling the coke drums, one would probably also have to take into account other reactions such as the secondary order decomposition reactions of aromatics to form coke.

Cracking velocity correlations for petroleum fractions as a function of boiling point and specific gravity are given by Hirsch and Fisher (1955), but these data were adjusted to match the heat balance of the furnace. Generally, the heaviest components have the highest cracking velocity. Heat of reaction data are based on estimating the heat of combustion for the hypothetical components by Winn's nomogram (1957).

The stoichiometry for the cracking is somewhat different for each hypothetical component and is estimated from plant data for the overall product distribution and by matching the carbon and hydrogen balance for each reaction. This stoichiometric model may be improved by taking into account additional feedstock parameters such as Conradson Carbon, ash content, viscosity etc.

\section{Phase equilibria}

The RKS equation of state (Soave 1972) is used to model the phase equilibria. Only one liquid phase is taken into account even though there is water present since the temperature at the injection point is higher than $T_{\mathrm{c}}$ for water.

Since temperature is important for both mass (Eq. 1) and momentum (Eq. 4) balances, it has to be calculated at each integration step from the enthalpy given by Eq. 3. An isenthalpic flash calculation using a cubic equation of state results in an implicit model with three nested iteration loops. It was decided to use an in-house implementation by Gundersen (1982), which has emphasis on the computation speed but lacks the robustness of general purpose methods.

The VLE calculations often have an important impact on the overall computation time in process simulations. Putting a rigorous 25 components VLE calculation inside the integration of $\left(n_{\text {hyp }}+1\right)$ coupled ordinary differential equations was a major concern at an early stage of the project. The advantage of using in-house software for the flash calculations is the ease with which special actions may be built into the program in order to reduce the computer time. This includes the storage of the previous solution as well as updating and storing the gradient of temperature with respect to reactor coordinate in order to get a good starting point for the next flash calculation. These actions have brought the time spent in the VLE calculations down from originally $65 \%$ to about $30 \%$ of the total computation time.

\section{Tube wall temperatures}

As the heat flux profile is given, heat balance calculations are not needed while integrating through the reactor. The total heat input may be adjusted in order to 
match the desired (or measured) outlet temperature. After the calculations have converged, the inner and outer tube wall temperatures are obtained by using the given heat flux profile together with the calculated temperatures on the process side. Equations (11-13) have been used in the simulation to obtain these temperatures.

$$
\begin{gathered}
T_{\mathrm{f}}=T+Q / h \\
T_{\mathrm{wi}}=T_{\mathrm{f}}+Q \cdot \delta / k_{\mathrm{c}} \\
T_{\mathrm{wo}}=T_{\mathrm{wi}}+Q \cdot d / k_{\mathrm{w}}
\end{gathered}
$$

The heat transfer coefficient $h$ is calculated according to Chen's method (1966) which gives approximately $2000-3000 \mathrm{~W} / \mathrm{K} \cdot \mathrm{m}^{2}$. Heat conduction through the coke layer (thickness $\delta$ ) and tube wall (thickness $d$ ) are also taken into account in order to calculate the outer tube wall temperature which is often limiting for the operation of the coker furnace. The thermal conductivity of coke $\left(k_{\mathrm{c}}\right)$ is a very uncertain parameter, and it varies depending on the type (age) of coke deposited inside the tubes.

\section{Coke deposition}

When describing the physical phenomena in the coker furnace, the coke deposition on the inner walls of the tubes was presented as a steady state between the chemical deposition of coke and the physical removal of this coke layer caused by the flow conditions in the tubes. The rate of deposition of coke on the walls depends on the composition and amount of cracked material, the temperature, and the flow characteristics in the tubes. Simple models have been presented for the chemical deposition of coke in thermal cracking processes (see for example Froment et. al. (1981)). Attempts were made to describe the removal of coke from the walls by simple models based on the shear stress acting on the walls of the tubes. These models predicted that coke deposition would be worst near the outlet of the furnace, where the temperature is highest. Since in reality the walls are almost clean at the end of the reactor, the removal of coke must depend on turbulent flow conditions, and first order models (shear stress) are insufficient to describe this process.

The coke deposition also depends on operating parameters which may not be included in a simulation model, such as hot spots resulting from local differences in the heat transfer and short time stops in the flow in one or more of the tubes. Based on this fact, we did not go into detail in obtaining a rigorous model of the deposition process. Rather, we calculated some 'coking factors' based on the simple steady state approach outlined above, which are useful for indicating the rate of coking.

\section{Feed characterization}

The basis for the characterization of the feed to the heater is a TBP curve and a density curve. The feed is divided into boiling point fractions which are treated as hypothetical components with fixed properties. The data for each of these hypothetical components are estimated using established methods from the API Data Book (1981). The data include $T_{\mathrm{c}}, P_{\mathrm{c}}$ and $\omega$ needed in the RKS equation to calculate the VLE behaviour in addition to molecular weight, ideal gas enthalpy (supplied as data to the program from PROCESS, mean boiling point, density, $\mathrm{C} / \mathrm{H}$ ratio and heat of combustion (estimated in the program).

In the simulation program, about 5 hypothetical components are used to describe the feed. Thirteen components are used to describe the light products. In 
addition, there is water and a hypothetical component which is supposed to represent coke. The light components currently used are $\mathrm{H}_{2}, \mathrm{H}_{2} \mathrm{~S}, \mathrm{CH}_{4}, \mathrm{C}_{2}, \mathrm{C}_{3}, \mathrm{C}_{4}$, $\mathrm{C}_{5}, \mathrm{C}_{6}, \mathrm{C}_{7}, \mathrm{C}_{8}, \mathrm{C}_{9}, \mathrm{C}_{11}$ and $\mathrm{C}_{13}$. The hypothetical components are supposed to crack directly to these light components and coke through a first order reaction.

The feed to the furnace is divided into hypothetical components based on the TBP curve of the feed. If this TBP curve is not available (as is the case when studying alternate feedstocks) an artificial TBP curve has to be constructed based on the light components being recirculated from the coke drums and the TBP data for the feed to the fractionation column.

The simulation model uses the general purpose flowsheet simulator PROCESS to supply component data as well as for characterization of the feed based on TBP and density data. This has been achieved by writing a 'user added subroutine' which is linked with PROCESS and which prints component data to a file in a format which is accepted by the coker furnace program.

\section{Thermophysical data}

As mentioned above, the flowsheeting program PROCESS is used to retrieve physical data for the components. Databank values are used for library components, while data $\left(T_{\mathrm{c}}, P_{\mathrm{c}}, \omega\right.$ and enthalpy coefficients) for the hypothetical components are estimated by the Lee-Kesler correlation (1975).

The enthalpies of both the liquid and vapour phase are calculated from ideal gas enthalpy and the non-ideal contribution from the RKS equation of state. While gas density is obtained from the RKS compressibility for the vapour phase, liquid density is based on methods recommended in the API Data Book. A computerized version of API method 6A3.5 for liquid density is included in Appendix 1 (O. Johnsen) as the API Data Book states that this nomogram is very difficult to computerize. This correlation has been found to be better suited for heavy fractions than the Lu method (1959).

The remaining physical properties (liquid viscosity and thermal conductivity, vapour viscosity) are estimated from methods in the API Data Book. Density and viscosity are used in pressure drop calculations. The Reynolds numbers (Re) in the coker furnace are in the order of $10^{6}$ to $10^{7}$. This means that the friction factor will be almost independent of Re and we expect the pressure drop to be almost independent of viscosity. This is confirmed by the sensitivity analysis which shows that the gas density is the most important physical property, followed by liquid density. Liquid viscosity, which is the most uncertain property, is fortunately of minor importance for the accuracy of the pressure drop calculations.

\section{Numerical solution}

Since the objective of this paper is to present an approach to solve a complex industrial 'unit operation' like a delayed coker furnace, rather than to discuss the optimal operation of such a unit, emphasis will be on program performance rather than calculated results for the process.

\section{Calculational strategy}

The $n_{\text {hyp }}+1$ coupled ordinary differential equations are solved by the variable order, variable step-length Hindmarsh-LSODE algorithm (Hindmarsh 1983). In 
LSODE there are implementations of the implicit Adams methods and the Backward Differentiation Formulas (BDF) in order to handle both nonstiff and stiff problems. For pressure drop calculations, the bends of the tubes are assigned equivalent tube lengths in order to let the ODE solver work continuously from the inlet to the outlet of the furnace. With 20 or 25 components taking part in the rigorous VLE calculations, and with 6 or 11 coupled ordinary differential equations to be solved for a reactor with a pressure drop of nearly 20 bars, it was considered possible that the resulting computer times would become prohibitive. After all, the calculation of the furnace is only the inner loop in a two-dimensional iteration (Newton-Raphson) to match outlet pressure and temperature, by varying inlet pressure and amount of heating in the furnace.

When considering the coker furnace as a tubular reactor, it is important to note that there are changes in the tube geometry (diameter, bends and elevation), which result in stepwise changes in the derivative of pressure with respect to reactor length. Another discrete phenomenon is the steam injection at a certain point in the furnace. At these points the integration will normally have to be restarted (initialize the Jacobian matrix), which is time-consuming. The heat flux profile has also been discretized over groups of tubes. Since, however, temperature is not a state variable solved by numerical integration, but rather obtained from isenthalpic flash calculations at each integration step, no special actions are needed.

Because of the discrete nature of the problem (pressure drop in bends, changes in diameter, steam injection), it was considered at an early stage to use the continuous stirred tank reactor (CSTR) approach, with one CSTR for each of the 55 tubes. However, by using equivalent tube lengths for bends in the pressure drop calcu-

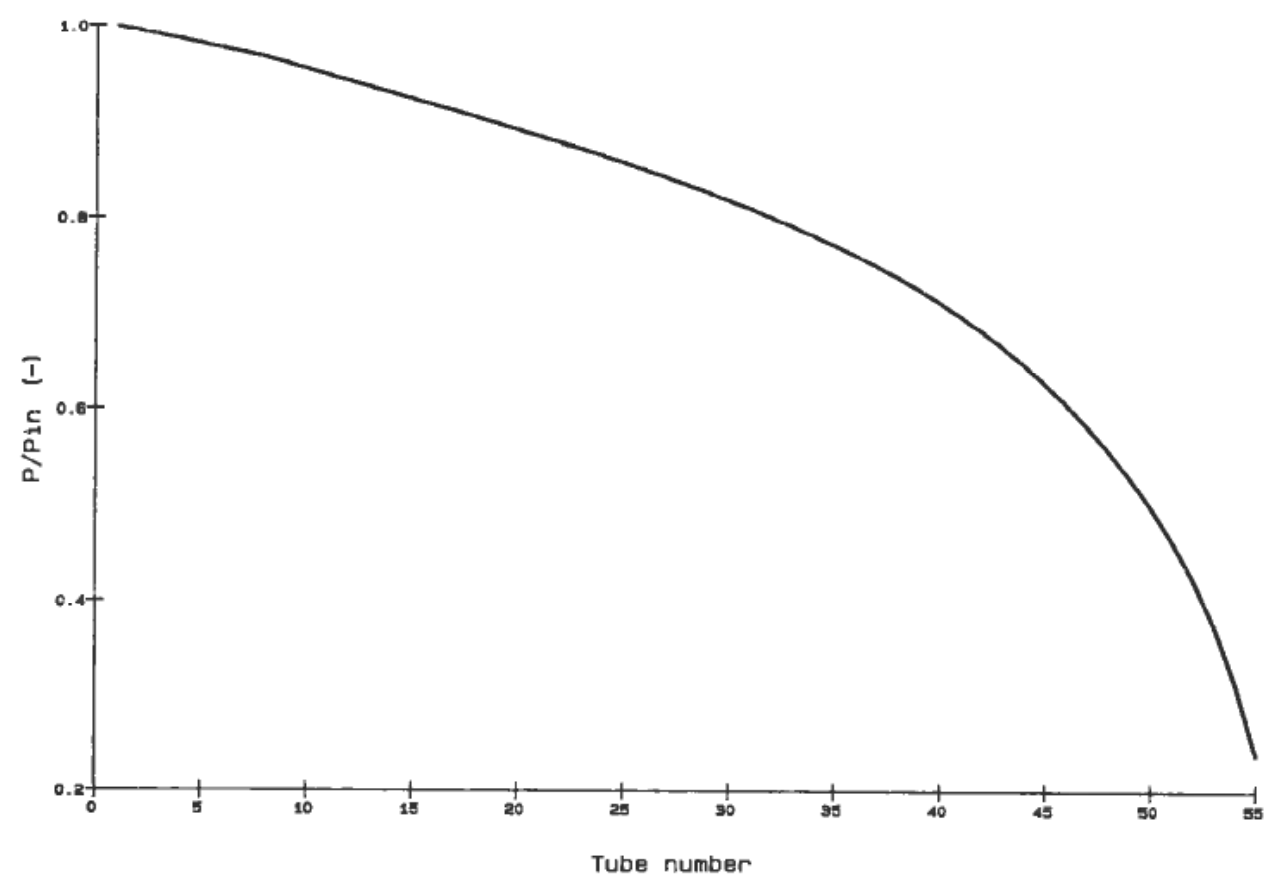

Figure 3. Pressure profile in the coker furnace. 


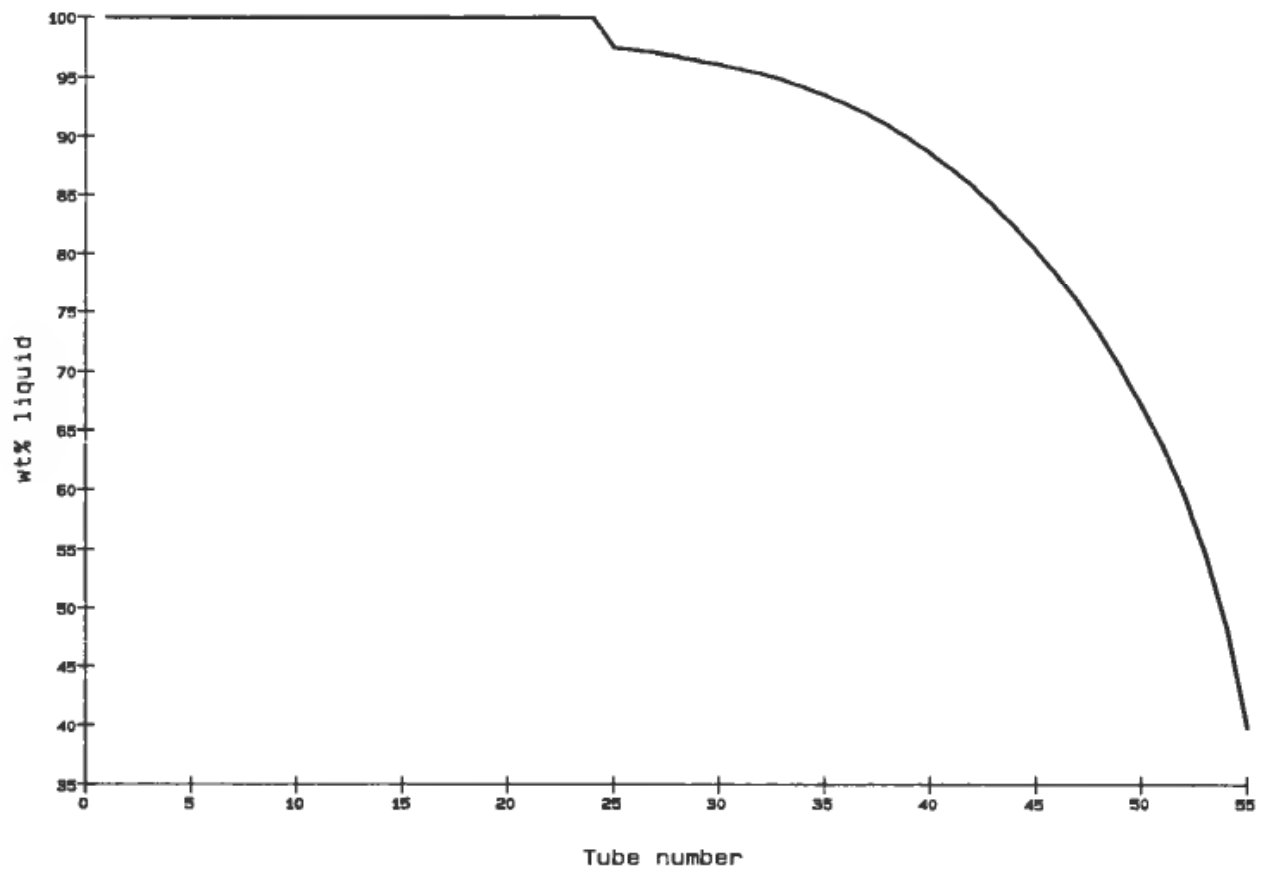

Figure 4. Liquid fraction profile in the coker furnace.

lations, the problem was reformulated and solved using the significantly more efficient and accurate differential equation approach.

While state variables do not change much during the first tubes in the furnace, they accelerate near the outlet (see Fig. 3 and Fig. 4 showing the pressure and vapour fraction profile in the furnace). The CSTR approach would loose accuracy in the last part of the furnace, which coincides with the position for coke deposition.

Fortunately, the computer times were not prohibitive when using the tubular reactor approach. With 5 hypothetical components (giving a total of 20 components), the total CPU-time is about 4 minutes. The furnace is typically calculated 8-10 times in order to solve the two-point boundary value problem, which means that each calculation of the reactor takes less than 30 seconds. While computer times were nearly doubled when using 10 , rather than 5 , hypothetical components, the effect on the simulation was negligible and 5 hypothetical components seem sufficient to describe the chemical reactions taking place. These figures relate to an implementation on a UNIVAC-1100/62.

There are two important reasons why computer times are as short as shown above. The first is the fact that the LSODE algorithm is able to take advantage of the nature of the actual problem. In the first part of the furnace, the gradients are nearly constant and LSODE is able to take up to ten tube lengths in one integration step. The second is the advantage of using in-house software for VLE calculations. This software may not show the robustness of general purpose routines, but is usually correspondingly faster. It is also easy to make special actions related to saving previous values of iterative variables and predicting new starting values by using stored gradient information. 


\section{Computational details}

The most important gradient which is stored during execution is the temperature gradient $d T / d z$. At each integration step, an isenthalpic flash calculation has to be carried out. Since this calculation, when using a cubic equation of statc, results in three nested loops (one for temperature, one for vapour fraction, and one for compressibility), the impact on the total CPU-time is significant. One problem that one faces when using automatic routines for solving the differential equations, is the unpredictable pattern of $z$ values for which the right hand side is calculated. Storing $d T / d z$ overcomes this problem and makes it possible to come up with a good estimate for temperature.

One way of establishing an initial estimate for the temperature is to use the value from the previous integration step. Experience showed, however, that three to five outer loop iterations were necessary to converge the isenthalpic flash calculation, and the resulting computer times were disappointing. Even with fairly good initial estimates, if the first calculation is not accepted, the second is only a predescribed adjustment of the independent variable (temperature) and three outer loop iterations are usually needed. When storing $d T / d z$, it is possible to estimate the temperature with an accuracy often accepted in the first iteration by the numerical routine for the isenthalpic VLE. The difference between one and three to five iterations is obviously significant for the total computer time needed to run the model. While VLE calculations in process simulations often exceed $70 \%$ of the overall computer time, the special actions described above halved the total CPU time and the time spent in the VLE routines was brought down to about $30 \%$ of the total computation time.

Results at specified points in the reactor (e.g. the inlet or outlet of each tube) are obtained by interpolation. There is no control over the integration itself which may proceed beyond the end of the reactor. Care must thus be taken to avoid things 'blowing up' numerically if this happens. As the pressure gets low, liquid evaporates and the vapour and liquid velocity increase rapidly in order to maintain the constant mass flow. This acceleration needs a high pressure drop as driving force, the result being a rapidly increasing pressure drop.

It may well be physically impossible to get the given mass flux through the reactor with the given inlet pressure. If the calculated pressure in a certain tube gets below a given criterion, the computer program stops the integration and restarts with an increased inlet pressure. In addition to the obvious problems involved in transferring negative pressure values to the physical property routines, one could also run into excessive computer times because of the stiff nature of the problem as the pressure gets low and the corresponding acceleration gets high.

In order to match outlet temperature and pressure by varying inlet pressure and amount of heating, a two-dimensional search must be done, since the flow of information is the opposite of the flow of mass through the furnace (two-point boundary value problem). Since temperature and pressure are highly coupled in the actual unit (e.g. through the vaporization of the liquid phase through the unit), a simultaneous (Newton-Raphson) iteration was chosen rather than the nested loop approach. The $2 * 2$ Jacobian matrix is found by numerical differentiation, and is updated after five iterations through the furnace exclusive of the iterations that are made to establish the matrix. Five iterations were chosen, since the calculation often converges after a total of seven or eight iterations, and thus only one evaluation of the Jacobian is needed to obtain the solution. 


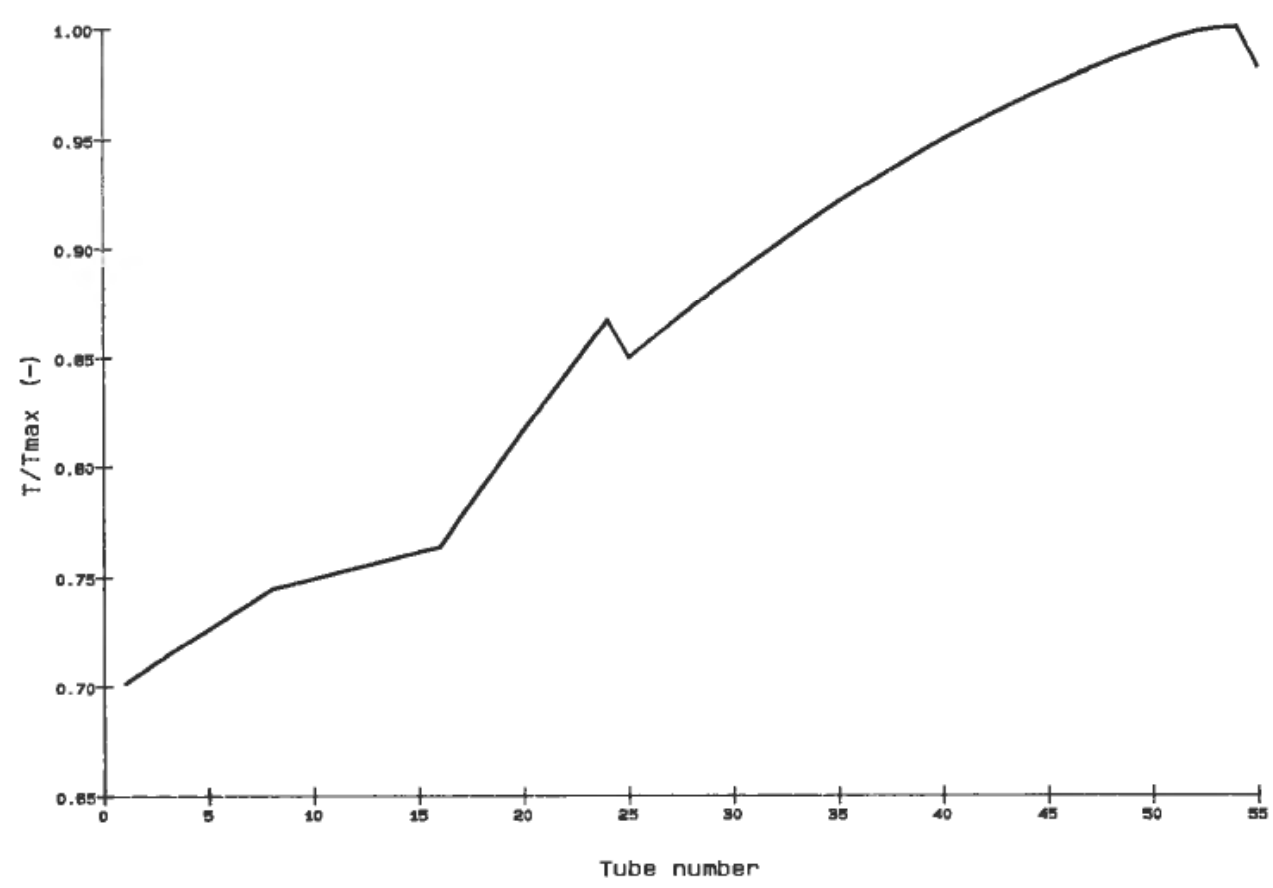

Figure 5. Temperature profile in the coker furnace.

\section{Use of the program}

Input data for the computer program include geometric data for the tubes, heat flux data, thickness of coke layer, steam injection data and inlet and outlet temperature and pressure. In addition there will be a separate file containing all the component data. Since the KOKSOVN program does not have its own data base, this file is generated by using PROCESS. Furthermore there will be a file with stoichiometric and kinetic data for the cracking reactions. Typical simulation results are shown in Figs. 3-5.

\section{Conclusion}

The computer program has proved to be predictive for the case where a feed characterization is available. Although the chemical reaction has been modeled with simplified methods (first order kinetics and total reactions only), the other phenomena such as two-phase flow pressure drop and vapour liquid equilibrium are rigorously modeled (compositional VLE). Cracking velocity has been used as an adjustment factor to match heat balance data.

When simulating other feedstocks, with only TBP data for the fresh feed available, the agreement with plant data is not as good. The reason for this is that the feed to the furnace (given as a TBP curve) is actually made up from the fresh feed to the distillation column upstream of the furnace and the light fractions recirculating from the coke drums. To be able to simulate the effects of other feedstocks, it seems necessary to include the coke drum and the bottom section of the column in the model. 
The computer times referred above are quite reasonable and shows that the rigorous approach taken in the modeling did not cause excessive computer times.

The acceleration pressure drop (due to increase in kinetic energy) is often neglected in two-phase pipeline programs because it is difficult to handle. The approach taken in this work, based on numerical differentiation is simple and seems to offer sufficient accuracy.

Another discussion often encountered in two-phase pipeline calculations is whether one should calculate the VLE behaviour at each integration step or use a preestablished table of values from which interpolated values may be obtained. For the actual problem, however, each chemical reaction adds a new dimension to this table of values, and with five cracking reactions (five pseudo components), the tabular approach is prohibitive.

Since a modular approach has been used in the development, the resulting computer program is a potential simulation tool for any non-adiabatic plug flow reactor with two-phase flow. The routines for thermophysical and transport properties, phase equilibria and chemical reaction may be replaced by other methods depending on the actual chemical system.

The program could also be used to simulate a pipeline with one or two-phase flow. Since, however, the total composition of the system in that case is constant (no chemical reaction), one could use preestablished tables for combinations of pressure and temperature, rather than performing tedious VLE calculations along the pipeline.

\section{Acknowledgements}

The authors wish to express their gratitude to the operating company Rafinor A/S \& Co., for the assistance and feedback during the development of the model, and also for the permission to publish this work. Acknowledgement is also given to our colleague Knut Harg whose earlier work on heat transfer and pressure drop in the furnace made things easier for us.

\section{Nomenclature}

$A_{\text {liq }}$ liquid void fraction in the tube

$b$ parameter defined in Eq. 6

$C_{\mathrm{c}} \quad$ parameter used in Eq. 9

$D$ inner tube diameter

$d$ differential operator or wall thickness (Eq. 13)

$F_{\mathrm{i}} \quad$ molar flow of component $\mathrm{i}$

$G$ mass flux

$g \quad$ acceleration of gravity

$H$ enthalpy

$h \quad$ film transfer coefficient

$i$ loop index (summation)

$k_{\mathrm{c}} \quad$ thermal conductivity of coke

$k_{\mathrm{w}} \quad$ thermal conductivity of the tube wall

$n_{\text {hyp }}$ total number of hypothetical components

$n_{\mathrm{r}} \quad$ number of independent chemical reactions

$P$ pressure 
$P_{\mathrm{c}} \quad$ critical pressure

$\Delta P$ pressure drop

$Q$ heat flux

$\operatorname{Re}$ Reynolds number

$r_{i} \quad$ rate of reaction for cracking reaction $\mathrm{i}$

$T$ temperature on the process side

$T_{\text {c }} \quad$ critical temperature

$T_{\mathrm{f}} \quad$ film temperature

$T_{\mathrm{o}} \quad$ temperature outside the tubes

$T_{\mathrm{wi}}$ inner wall temperature

$T_{\text {wo }}$ outer wall temperature

$U$ overall heat transfer coefficient

$x \quad$ weight fraction vapour ('quality')

$y$ vertical coordinate in furnace

$z \quad$ axial coordinate of the reactor

\section{Greek symbols}

$\alpha \quad$ cross section in the tubes occupied by vapour ('void fraction')

$\delta \quad$ thickness of coke layer

$\partial \quad$ partial derivation

$\rho$ density

$\omega$ acentric factor

\section{Subscripts}

a acceleration

c critical property

f friction

G gas phase

g gravitation

i loop index and component identifier

L liquid phase

r reaction

\section{REFERENCES}

API TeChnical Data BooK, vol. 1, 4th edition, 1983.

ASPEN TECHNOLOGY 'ASPEN PLUS Introductory Manual', Cambridge, Massachusetts, Dec. 1983.

Chemshare Process Systems Ltd. 'Guide to Solving Process Engineering Problems by Simulation', DESIGN/2000, Chemtran and Refine Manual, Wilmslow, England, March 1981.

CHEN, J. C. (1966). 'Correlation for boiling heat transfer to saturated fluids in convective flow', Ind. \& Eng. Chem., Proc. Des. Dev., 5, 3, 322-329.

Fletcher, P. (1983). 'An introduction to delayed coking', The Chemical Engineer, pp 21-23, Sept.-Oct. 1983.

GUNDERSEN, T. (1982). "Numerical aspects of the implementation of cubic equations of state in flash calculation routines', Comp. and Chem. Eng., vol. 6, no. 3, pp 245-255.

Hindmarsh, A. H. (1983). 'ODEPACK, a systematized collection of ODE solvers', in Stepleman, R. et al. (eds.) 'Scientific Computing', IMACS/North-Holland Publ. Co., pp $55-64$. 
HiRsch, J. H. and Fisher, E. K. (1955). 'Conditions and results of thermal cracking for gasoline', in Reinbolt 'The Chemistry of Petroleum Hydrocarbons', vol. 2, 27-60.

LEE, B. I. and KeSLER, M. G. (1975). 'A generalized thermodynamic correlation based on three-parameter corresponding states', AIChE J., 21, 3, 510-527.

Lu, B. C.-Y. (1959). 'Estimate specific liquid volumes', Chem. Eng., 66, 9, 137-138.

Simulation SCIENCES INC. 'PROCESS-Steady State Heat and Mass Balance Flowsheet Simulator', Input Manual, Rev. 3, Fullerton, California, Sept. 1983.

SoAve, G. (1972) 'Equilibrium constants from a modified Redlich Kwong equation of state', Chem. Eng. Sci., vol. 27, pp 1197-1203.

Whalley, P. B. (1981). 'Correlation for frictional two-phase pressure drop in uniform channels', in HTFS Handbook, vol. 3, Harwell and National Engineering Laboratory.

WinN, F. W. (1957). ' Physical Properties by Nomogram', Petroleum Refiner, 36, 2, 157-159.

Butterworth, D. and Hewitt, G. F. (ed.) (1977). 'Two-Phase Flow and Heat Transfer', Oxford Univ. Press.

Sundaram, R. M., Van Damme, P. S. and Froment, G. F. (1981). 'Coke Deposition in the Thermal Cracking of Ethane', AIChE J., 27, 6, 946-951.

\section{Appendix 1}

\section{Density of Petroleum Fractions \\ Calculator program for API method 6 A3.5}

by Oddvar Johnsen

Figure 6A3.5 in the API Data Book for density of petroleum fractions at atmospheric pressure has been computerized through the development of a set of equations. A program for the HP-41C calculator is available on request from the author.

The method is valid for a limited density range and is based on the Watson $\mathrm{K}$ correction. The method was developed for calculating the density of heavy petroleum fractions (atmospheric residue) at high temperatures based on density measurements at $15^{\circ} \mathrm{C}$. The equations involved are:

$$
\begin{aligned}
L & =a_{1}+a_{2} \cdot \rho_{15}+a_{3} \cdot \rho_{15}^{2}+\left(a_{4}+a_{5} \cdot \rho_{15}+a_{6} \cdot \rho_{15}^{2}\right) \cdot\left(a_{7}+a_{8} \cdot K+a_{9} \cdot K^{2}\right) \\
\alpha & =a_{10}+a_{11} \cdot \rho_{15}+a_{12} \cdot \rho_{15}^{2} \\
Y_{\rho} & =a_{13}+a_{14} \cdot \mathrm{T}-\left(a_{13}+a_{14} \cdot T-L \cdot \sin \alpha\right) \cdot 135 /(L \cdot \cos \alpha) \\
\rho_{\mathrm{T}} & =a_{15}+a_{16} \cdot Y_{\rho}+a_{17} \cdot Y_{\rho}^{2}+a_{18} \cdot Y_{\rho}^{3}
\end{aligned}
$$

where

$$
\begin{array}{ll}
K & =\text { Watson } K \\
T & =\text { Temperature }\left({ }^{\circ} \mathrm{C}\right) \\
\rho_{15} & =\text { Relative density } 15^{\circ} \mathrm{C} / 15^{\circ} \mathrm{C}\left(\mathrm{kg} / \mathrm{dm}^{3}\right) \\
\rho_{\mathrm{T}} & =\text { Density at given temperature }\left(\mathrm{kg} / \mathrm{dm}^{3}\right) \\
L, \alpha, Y_{\rho} & =\text { For intermediate calculations only }
\end{array}
$$

Constants obtained by parameter estimation are as follows:

$$
\begin{array}{rr}
a_{1}=0 \cdot 32308700 \mathrm{E}+2 & a_{6}=-0 \cdot 94260000 \mathrm{E}-1 \\
a_{2}=-0 \cdot 10745340 \mathrm{E}+3 & a_{7}=0 \cdot 36273770 \mathrm{E}+1 \\
a_{3}=0 \cdot 19651380 \mathrm{E}+3 & a_{8}=0 \cdot 72666424 \mathrm{E}+2 \\
a_{4}=-0 \cdot 17419900 \mathrm{E}+2 & a_{9}=-0 \cdot 60605916 \mathrm{E}+2 \\
a_{5}=0 \cdot 26509000 \mathrm{E}+1 & a_{10}=-0 \cdot 14625400 \mathrm{E}+3
\end{array}
$$




$$
\begin{aligned}
& a_{11}=0.31904150 \mathrm{E}+3 \quad a_{15}=0.55091300 \mathrm{E}+0 \\
& a_{12}=-0 \cdot 11967640 \mathrm{E}+3 \quad a_{16}=0.37160000 \mathrm{E}-2 \\
& a_{13}=0.39600000 \mathrm{E}+0 \quad a_{17}=-0 \cdot 10760000 \mathrm{E}-4 \\
& a_{14}=-0.59400000 \mathrm{E}+1 \quad a_{18}=0.22000000 \mathrm{E}-7
\end{aligned}
$$

With $\rho_{15}$ in the range $0 \cdot 7-1.0 \mathrm{~g} / \mathrm{cm}^{3}$, the results when using the method are obtained with an accuracy of $\pm 0.005 \mathrm{~g} / \mathrm{cm}^{3}$ relative to API figure $6 \mathrm{A3} .5$. The equations are not valid for $\rho_{\mathrm{T}}$ lower than $0.55 \mathrm{~g} / \mathrm{cm}^{3}$. 